\title{
Escuelas que tienen su propia voz. Experiencias de un proceso de autoevaluación en 30 instituciones educativas
}

Schools that have their own voice. Experiences of a self-evaluation process in 30 educational institutions

\section{RESUMEN}

Mgr. Roberto Barrientos

Mollo

Comunidades de aprendizaje roberto.b@comunidaddeaprendizaje.pe

Recibido

$10 / 10 / 2018$

Revisado

$10 / 11 / 2018$

Aceptado

$04 / 12 / 2018$
- I propósito de la investigación fue obtener una mejor - comprensión de los procesos de autoevaluación escolar para lo cual se buscó comprender las vivencias y percepciones de los principales actores involucrados en un proceso de autoevaluación institucional. En este se ofreció la posibilidad de una construcción participativa del modelo de escuela, así como los indicadores e instrumentos de evaluación de la misma. Dicho proceso de autoevaluación tuvo una duración de dos años e involucró a 30 escuelas de diferentes niveles educativos. La información se recabó a partir de una encuesta y revisión documental. Los resultados del estudio mostraron que las personas están abiertas a colaborar y ser parte de procesos de autoevaluación y, además, que es necesaria la figura de un apoyo externo para el desarrollo de capacidades. También se descubrió que el incentivo económico es un elemento que puede contribuir para la adopción inicial; sin embargo, se corre el riesgo de generar dependencia de dicha motivación extrínseca.

Palabras clave: autoevaluación escolar, eficacia escolar, rendición de cuentas, mejoramiento escolar, cambio educativo. 


\section{ABSTRACT}

The purpose of the research was to obtain a better understanding of the processes of school self-evaluation. To this end, an attempt was made to understand the experiences and perceptions of the main actors involved in an institutional self-evaluation process in which the possibility of a participative construction of the school model was offered, as well as the indicators and evaluation instruments of the same. This self-assessment process lasted two years and involved 30 schools of different educational levels and had the presence of a facilitator and an economic incentive from the regional government. The information was gathered from a survey and documentary review. The results of the study showed that people are open to collaborate and be part of self-evaluation processes. Furthermore, the figure of external support for the development of capacities is necessary. It was also discovered that the economic incentive is an element that can contribute to the initial adoption, however there is a risk of generating dependency on this extrinsic motivation

Keywords: school selfevaluation, school effectiveness, accountability, school improvement, educational change. 


\section{INTRODUCCIÓN}

a educación es, hoy en día, un tema de gran interés social, la cual ha generado diversas iniciativas para la mejora de los procesos educativos. La autoevaluación escolar es una de ellas. La experiencia en procesos de autoevaluación escolar en el Perú es todavía incipiente, por lo que es importante generar aportes al respecto. El presente estudio permite comprender las reacciones de las escuelas frente a los proceso de autoevaluación para tenerlas en cuenta en futuros procesos del mismo tipo. Desde una perspectiva teórica, el presente estudio contribuye al cuerpo teórico de procesos de evaluación interna escolar en el país y es su propósito obtener una mejor comprensión de los procesos de autoevaluación escolar.

\section{La rendición de cuentas}

Son múltiples los estudios que buscan comprender cuál es el efecto que logra tener la escuela en la vida de los estudiantes (Baudelot \& Leclercq, 2005). Un movimiento que tiene cada vez más fuerza a nivel mundial es la rendición de cuentas o accountability con sus respectivos impulsadores y detractores (Scheerens, 2007; Supovitz, 2009). La naturaleza del mismo consiste en exigir a la escuela que haga públicos sus procesos y resultados. En la línea econométrica, están los estudios de tasas de retorno centrados en correlacionar aspectos determinados de la formación de un individuo, como son las certificaciones logradas y logros en evaluaciones estandarizadas de lectura y matemáticas (E. Hanushek, 1995; E. A. Hanushek, 2002; Psacharopoulos \& Patrinos, 2004). Últimamente, se ha tratado de agregar al análisis las habilidades sociales 0 habilidades blandas (Heckman, 2011), por lo que se puede afirmar que la rendición de cuentas está presente en el debate de las políticas públicas y que en educación se ha centrado exclusivamente en la mejora de rendimiento en evaluaciones estandarizadas en áreas básicas como lectura y matemáticas.

\section{La investigación en eficacia escolar}

El informe de John Coleman (1966) concluyó que la escuela no hacía la diferencia. Este estudio generó el inicio de una serie de investigaciones en respuesta al mismo que darían a luz, años después, al Movimiento de Eficacia Escolar (School Effectiveness). Así mismo, se fueron evidenciando las deficiencias metodológicas y de enfoque del mismo (Creemers \& Reezigt, 2005; Jaap Scheerens, 1999; 2000; Scheerens \& Bosker, 1997). Estos estudios demostraron que la escuela sí hace la diferencia. La 
investigación de eficacia escolar ha establecido ciertos elementos característicos de las buenas escuelas. Una larga lista de factores o indicadores de calidad educativa se han generado en diversas partes del mundo. Existen propuestas que van desde una amplia gama de indicadores como el de Scheerens y Bosker (1997) de cerca de 700 indicadores a más simples como el de Pam Sammons (1995b). En su libro Key Characteristics of Effective Schools (1995a), Sammons resume en 11 los factores de eficacia escolar:

1. Metas y visión compartidas

2. Un ambiente de aprendizaje

3. Refuerzos positivos

4. Focalización en los procesos de enseñanza-aprendizaje

5. Seguimiento del progreso

6. Una organización del aprendizaje

7. Liderazgo profesional

8. Relaciones escuela-familia

9. Enseñanza con propósito

10. Altas expectativas frente a los estudiantes

11. Normas de convivencia claras

Después de tantos años de investigación, en la escuela existen acuerdos comunes sobre los factores que hacen a una escuela eficaz.

\subsection{El proyecto piloto de evaluación de la calidad escolar}

El proyecto piloto de evaluación de la calidad escolar es una iniciativa de la Comisión de Educación de la Unión Europea, la cual consistió en promover procesos de evaluación de la calidad escolar en los países miembros. Es así que se encomendó a un grupo de expertos la preparación de un proyecto de intervención con el objetivo de promover una cultura de evaluación de la calidad. Los investigadores seleccionados fueron John MacBeath, que en ese entonces trabajaba en la universidad de Strathclyde (Inglaterra); Denis Meuret, de la universidad de Borgoña (Francia); Michael Schratz de la Universidad de Innsbruck (Austria) y Lars Bo Jacobsen (Holanda). Todos ellos con antecedentes en autoevaluación y evaluación de la calidad educativa en sus respectivos 
países. Sin embargo, quien había realizado un trabajo más innovador sobre el tema evaluativo era Macbeath. Por lo que la propuesta a la Comisión de Educación de la UE estuvo basada en las experiencias previas realizadas por Macbeath, en Escocia, que fomentaban la autoevaluación escolar en vistas a un fortalecimiento de la autonomía escolar y autonomía profesional docente. A continuación, se desarrollan los trabajos de Macbeath y luego la propuesta que se realizó para la Comisión Europea.

\section{El Proyecto de autoevaluación escolar de MacBeath}

Es en el contexto inglés de los años 90, caracterizado por constantes evaluaciones externas y de pocos espacios para la autonomía evaluativa escolar, que MacBeath reflexiona sobre la importancia de ofrecer mayores espacios para fortalecer la autonomía escolar. Una expresión de esta autonomía es un proceso de autoevaluación escolar como una idea de calidad construida participativamente. Esto es que la escuela decida revisarse ella sola y, además, con la participación de toda la comunidad educativa en los tópicos que ellos decidan y no los impuestos por los órganos de control del gobierno. La escuela construye su propia idea de calidad y lo hace de manera participativa (Castoldi, 2001). Estas acciones iban en la línea de los estudios y desarrollos teóricos de investigación-acción con mayor presencia en ese momento con autores icónicos como Eliot (1981) y Stenhouse (1980), y, desde fuera de Inglaterra, autores como el australiano Kemmis (Kemmis \& Robin McTaggart, 1988; Kemmis \& McTaggart, 1988; Kemmis \& Wilkinson, 1998). Para Macbeath "un signo de buen estado de salud de un sistema educativo lo vemos cuando sus escuelas tienen un alto grado de inteligencia organizacional y un know how acerca de cómo usar herramientas autoevaluativas para el auto-mejoramiento" (Macbeath, 1999, p. 1). En los años noventa, empieza a desarrollar una metodología para involucrar a toda la escuela. Luego de los resultados positivos de la misma, el Sindicato Nacional de Profesores (National Union Teachers) le encargó en el año de 1995 elaborar una propuesta para toda la nación y probar su validez ${ }^{1}$. Es así que el autor fue desarrollando una metodología que permite a directores, docentes, alumnos y padres evaluar su propia escuela. Este modelo debería de ser suficientemente sólido y flexible para adaptarse a una multitud de contextos si quiere ser fiel a su idea original de ayudar a narrar la "historia" única de cada escuela. En 1995, se seleccionan 10 escuelas de Inglaterra y Gales para un trabajo de validación de la propuesta (Tabla 1 y 2). Al respecto, se

1. Recientemente la asociación mundial de sindicatos, International Education, le encargó la elaboración de un estudio sobre el futuro de la educación: MacBeath, J. (2012). Future of the teaching profession. International Education \& University of Cambridge. 
generaron encuentros con los actores de la escuela (profesores, alumnos, padres, directores, personal de apoyo, consultores), invitándolos a identificar los criterios que hace a una escuela eficaz. Después de haber analizado los indicadores, en función a su especificidad y su grado de prioridad para cada actor; los investigadores del grupo de Macbeath han formado grupos de indicadores relacionados a ciertos temas y así sucesivamente han formado un esquema general que hizo coincidir las diversas preocupaciones de los diversos actores invitados. La equidad, el apoyo durante el proceso de aprendizaje y el clima en la clase son preocupaciones que emergieron de los alumnos; mientras que el apoyo a la enseñanza, la organización, la comunicación y la relaciones entre la escuela y la familia son temas que emergieron de los adultos. Otros cuatro temas surgieron tanto en grupos de adultos como de niños: el tiempo como recurso, el clima de la escuela, las relaciones y el reconocimiento del éxito.

Tabla 1

Número de escuelas participantes

\begin{tabular}{lr}
\hline Primaria & 6 \\
Secundaria & 3 \\
Especial & 1 \\
\hline Total & 10 \\
\hline
\end{tabular}

Tabla 2

Participantes a la experiencia

\begin{tabular}{lcc}
\hline \multicolumn{1}{c}{ Distrito } & Primaria/Especial & Secundaria \\
\hline Profesores & 48 & 61 \\
Directivos & 10 & 16 \\
Administrativos & 19 & 15 \\
alumnos & 201 & 181 \\
Padres & 67 & 31 \\
Miembros del gobierno & 24 & 29 \\
\hline Total & 369 & 333 \\
\hline
\end{tabular}


En base a dicha experiencia, el paso siguiente fue generar una ficha que tenga las dimensiones que han sido discutidas o pedidas por la mayoría, llamado un perfil de autoevaluación. "¿Por qué un modelo distinto para cada una? Porque las escuelas son diversas cada una. "Las escuelas deben hablar por sí mismas" esta afirmación presenta en el fondo tres preguntas sencillas que debe hacer toda escuela: "¿Qué estamos haciendo bien? ¿Qué podemos mejorar? ¿Quién necesita ayuda?" (Macbeath, 1999, p. 60)

En la experiencia de Escocia los elementos a lo que se invitaba a la escuela a autoevaluarse en el perfil de autoevaluación fueron:

- Clima escolar

- Relaciones

- Organización y comunicación

- Uso del tiempo y recursos

- Reconocimiento de los logros

- Equidad

- Relación escuela hogar

- Apoyo para la enseñanza

- Clima de aula

- Apoyo para el aprendizaje

En este proyecto cada escuela construyó los elementos que, en su opinión, son parte de una escuela de calidad, realizando sus respectivos planes de mejora para las áreas seleccionadas.

\subsubsection{El proyecto Puentes a través de los límites}

Es en base a la propuesta anterior, es que se desarrolla el proyecto para los países de la Comunidad Europea que realizó algunas modificaciones por la magnitud; por ejemplo, se eliminaron los talleres de debate para construir las características de una buena escuela a partir de la que se elaboraba el perfil de autoevaluación. En vez de ello, se entregó directamente a cada escuela una ficha con 12 elementos previamente elaborados por los investigadores. Habría tomado mucho tiempo iniciar la construcción de los indicadores desde la escuela como fue en el proyecto original. El 
proyecto inició en el año 1996 con la participación de 114 escuelas secundarias de 14 países de con el nombre Puentes a través de los límites (Bridges across boundaries)². El proyecto tuvo tres elementos: una guía de autoevaluación con 30 instrumentos propuestos, una ficha de autoevaluación, llamado Perfil de autoevaluación, con 12 áreas para la revisión y un asesor externo, llamado amigo crítico. Los resultados del trabajo realizado en el año son recogidos en el Informe final Evaluación de la calidad de la escuela. Un proyecto piloto europeo ${ }^{3}$ (Macbeath, Schratz, Meuret, \& Jakobsen, 1999), donde se evidencian los resultados positivos de la experiencia. Más adelante dichos autores elaboraron una versión para la divulgación del proyecto en un libro titulado Autoevaluación en escuelas europeas: una historia de cambio (John MacBeath, Schratz, Meuret, \& Jakobsen, 2001). Posteriormente, fueron invitados a compartir la experiencia en diversos eventos representativos del mundo educativo como el Congreso Internacional de Eficacia y Cambio en Educación (ICSEI) (Swaffield, 2002, 2004). En el 2004, el proyecto fue asumido por el proyecto Comenius ${ }^{4}$. La experiencia continuó realizándose hasta el año 2007, y una reciente síntesis del mismo ha sido realizada por Francesca Brotto (2011). La experiencia dio lugar a múltiples iniciativas que han sido estudiadas por diversos investigadores (Alvik, 1996; Nevo, 1995).

\section{Elementos del proyecto}

\section{Los elementos son los tres siguientes:}

1. El Perfil de Autoevaluación (Figura 4.A) es una ficha de evaluación que presenta doce áreas de la vida escolar, tomadas de los factores de éxito que muestran los estudios de eficacia escolar (ver tabla 3: la familia, la comunidad, el trabajo, la calidad de los aprendizajes, el Apoyo en caso de dificultades, el tiempo para el aprendizaje, la escuela como lugar social, la escuela como espacio de desarrollo profesional. Los objetivos del perfil son:

Promover la discusión participada entre todas las personas y grupos de interés de la escuela, poniendo las bases para una cultura del análisis, de la reflexión y de la evaluación como proceso continuo. Obtener una imagen de la escuela vista desde diversas perspectivas: alumnos, profesores, padres y personal administrativo. Ayudar a identificar las áreas que posteriormente se verán

2. El nombre oficial del proyecto fue Evaluating Quality in School Education (EQSE).

3. Evaluating quality in school education A European pilot project, Final report. 
sometidas a una evaluación más exhaustiva, las áreas de necesidad (MacBeath, et al., 2001, p. 80).

El perfil es utilizado en uno o varios talleres de discusión en los que participan representantes de toda la comunidad educativa en grupos heterogéneos u homogéneos (en la Tabla 3.B se muestra el llenado de un perfil en una escuela del Callao).

Tabla 3. Ficha de Autoevaluación

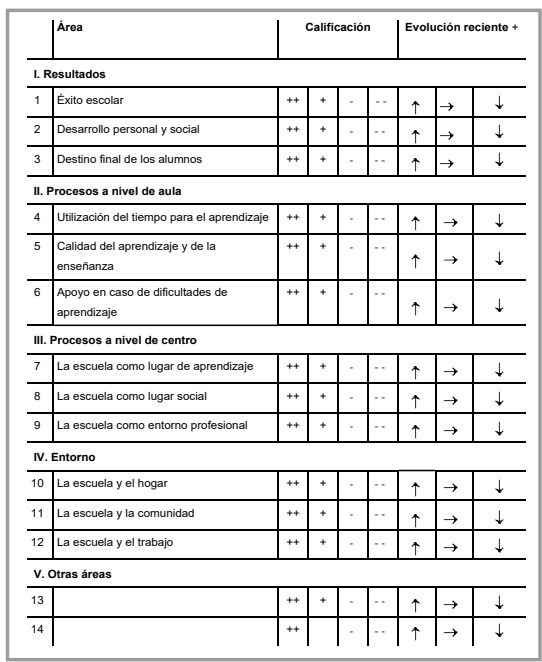

A. Perfil de Autoevaluación

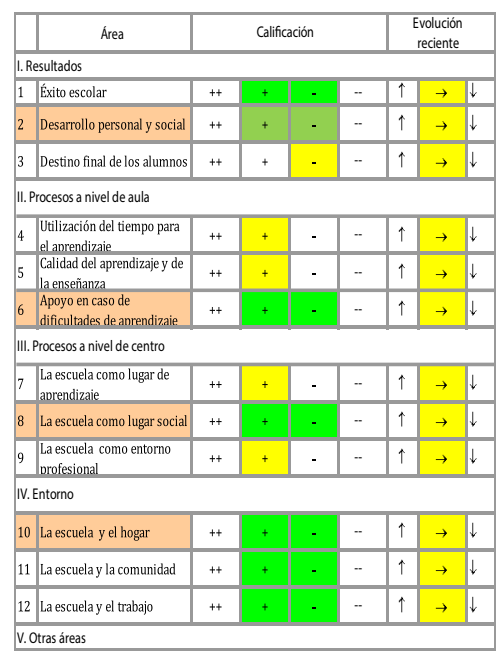

B. Ejemplo de la aplicación del perfil en una escuela

2. Una propuesta de métodos y técnicas de evaluación. Situadas en el enfoque cuantitativo y cualitativos (entrevista, campo de fuerzas, observación de pares, observación de alumnos, grupos focales, la sombra, etc.) para que sean aplicadas por las escuelas como medio de recolección de información después de haber seleccionado en los talleres del Perfil de Autoevaluación (PAE) 4 áreas a ser evaluadas.

3. El amigo crítico es un facilitador que acompaña a la escuela en el proceso. Al igual que un amigo ofrece a la escuela apoyo y confianza, al mismo tiempo, como un crítico es exigente, ofreciendo una perspectiva externa y que acompañará durante todo el proceso a las escuelas. 


\subsubsection{Descripción de la metodología}

El primer paso es la selección del ámbito o área que se quiere reforzar o mejorar. En segundo lugar viene la elaboración de parámetros de evaluación; luego, la construcción de indicadores y, por último, se selecciona las técnicas o instrumentos más aptos para las tareas programadas. La selección del área se realiza en un proceso participativo donde la característica más importante es la discusión participada entre todos los miembros de la comunidad: padres, alumnos, profesores, director, administrativos. Mediante el diálogo se llegan a acuerdos sobre una visión de la escuela para luego seleccionar 4 áreas a evaluar durante el año. Después se procede a elaborar los indicadores correspondiente, para luego construir instrumentos para la recolección de la información (Figura 1). El proceso termina con la implementación de los planes de mejora a lo largo del año.

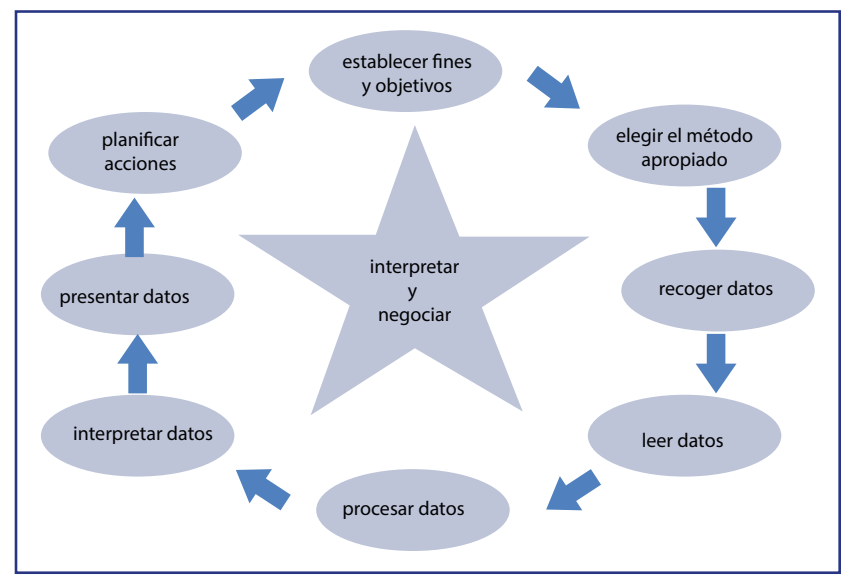

\subsection{El amigo crítico}

"La implicación externa es esencial para obtener el éxito" (Fullan, 2002, p. 212) por lo que en el proyecto estudiado se tuvo una figura del externo que acompañe a la escuela en el proceso de mejora. Un término que aparece en la literatura de cambio organizacional y mejora de aprendizajes en las escuelas es del amigo crítico o Critical Friend (Ainscow \& Southworth, 1996; Bambino, 2002; Costa \& Kallick, 1993; Cuban \& Shipps, 2000; MacBeath, 1998; Schein, 1997; Swaffield, 2002, 2004).

El término amigo crítico se viene usando desde los años setenta y se le atribuye a Desmond Nuttal quien desarrollara algunas experiencias autoevaluativas (Heller, 
1998). La figura del amigo crítico ha sido aplicada en diversos contextos. En el caso del Reino Unido, las Autoridades Educativas Locales (LEA en sus siglas en inglés) 5 las Autoridades de Inglaterra, estudios de apoyo a la escuela, procesos de autoevaluación escolar, programas de formación de directores, etc. y existen diversos estudios que analizan el rol de los colaboradores o asesores externos (Ainscow \& Southworth, 1996).

Según Costa y Kallik (1993) el amigo crítico es visto como

Una persona veraz que hace preguntas provocativas, provee información para examinarnos a través de otros lentes, y ofrece críticas del trabajo de las personas como un amigo. Un amigo crítico se toma el tiempo para comprender el contexto que presenta el trabajo y los resultados que la persona o el grupo están buscando lograr. El amigo es una persona que aboga por el éxito del trabajo. (p. 50)

En síntesis, el propósito de este "nuevo rol del amigo crítico en la evaluación es el proveer un contexto en el que las personas puedan recibir tanto críticas como soporte y apoyo para su trabajo"(Costa \& Kallick, 1993, p. 51). Esta es la esencia del trabajo del mismo para la mejora de la escuela. Como se puede ver no es un evaluador externo solamente, sino un acompañante y asesor al mismo tiempo. Tiene un rol clave en el cambio educativo al punto de "alertar a la escuela sobre temas que son sólo percibidos a medias"(Swaffield, 2004, p. 3) por la misma escuela. Este rol es percibido por muchos investigadores como bueno y necesario. Al respecto, Dean plantea la metáfora de la mirada, al decir que el amigo crítico es como "un par de ojos frescos" (Dean, 1992), o como "el oído atento", "el sintonizador", etc. Todas estas metáforas lo que buscan es mostrar que el amigo critico no solo es un extraño a la escuela sino que "también asiste y los apoya para ver lo familiar desde una nueva perspectiva" (Swafield, 2002, p.5). Swafield, quien ha realizado diversas investigaciones sobre el rol del amigo crítico, nos dice que para comprenderlo mejor es útil compararlo con otros roles como la supervisión, asesoría, mentores, tutores, coaches y consultores. Básicamente "un amigo crítico está preocupado por cuestiones de tipo organizacional y por los resultados, efectos e implicancias para diferentes tipos de personas, así como por el bienestar personal de los individuos" (Swaffield, 2004, p. 4). La relación de mentores, tutores y coaches es, siguiendo un análisis transaccional, de "padre-hijo"; mientras la del amigo crítico de "adulto-adulto". 
Por lo que, según la autora, el amigo crítico es alguien que presenta las siguientes características:

- Posee "licencia para ayudar"

- Está en una situación externa

- Construye y mantiene relaciones en torno a la verdad

- Es políticamente neutro

- Ofrece un respiro y una profundización acerca del conocimiento y experiencia relevante, hacia una situación específica.

- Establece o invita a focalizar esfuerzos y tender puentes para las tareas

- Balancea una amistad y la crítica, enfocado en un soporte personal y desafío profesional

- Motiva y consuela

- Es facilitador más que director

- Tiene a bien desarrollar una comprensión de las complejidades y procesos de cambio

- Es un defensor del éxito del trabajo (p.5).

El amigo crítico es una figura muy importante en el cambio educativo, pero "frecuentemente el arte de la crítica es pasada por alto en la vida de las escuelas"(Costa \& Kallick, 1993, p. 51). Es a tal punto importante que, dice Costa y Kallick, "introduciendo el rol de los amigos críticos en todos los niveles del sistema escolar construiremos una gran capacidad para la autoevaluación así mismo una apertura de mente para la construcción del pensamiento de los otros" (Costa \& Kallick, 1993, p. 51).

\subsection{La propuesta de autoevaluación de la región Callao}

Desde el gobierno regional del Callao, se decidió replicar la experiencia de Macbeath en 30 colegios pilotos con algunos componentes añadidos como: un acompañamiento psicopedagógico a las escuelas y un incentivo económico a los docentes por el trabajo extra realizado. El incentivo económico fue 100 nuevos soles mensuales durante la experiencia. Los fondos los otorgaba el Consejo de Administración del Fondo Educativo del Callao (CAFED). 
En la Figura 2 se muestran dos fotografías de la primera etapa de llenado del perfil de autoevaluación con toda la comunidad educativa: padres de familia (figura 2.A) y estudiantes (figura 2.B).

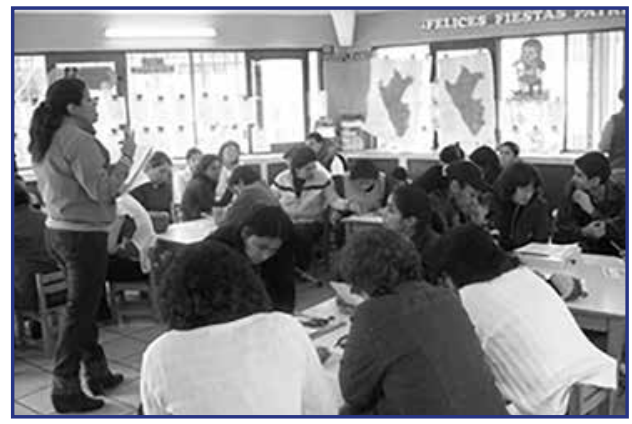

A. Taller con padres de familia

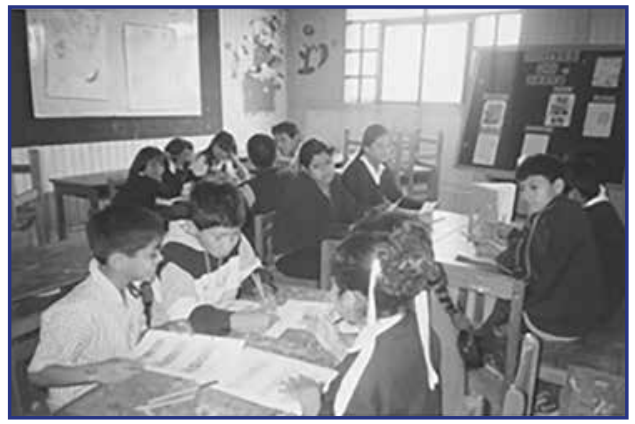

B. Taller con alumnos

Figura 2. Aplicación del Perfil de Autoevaluación en una Institución educativa

En la Figura 3 se muestra una de las acciones de sensibilización acerca del proceso en las escuelas. Todas las escuelas realizaron un periódico mural con diversas temáticas de periodicidad mensual sobre el proyecto

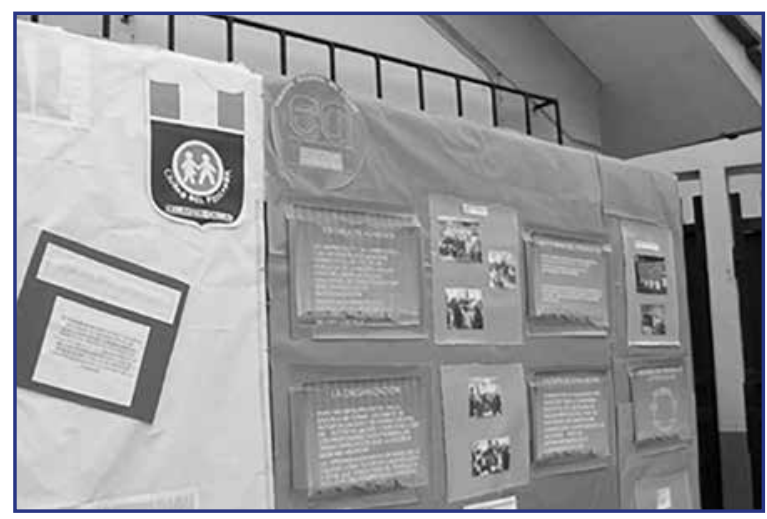

Figura 3. Acciones de sensibilización en las escuelas. 
El comité de calidad tuvo entre sus funciones la elaboración de los instrumentos de autoevaluación, como se observa en la Figura 4. .

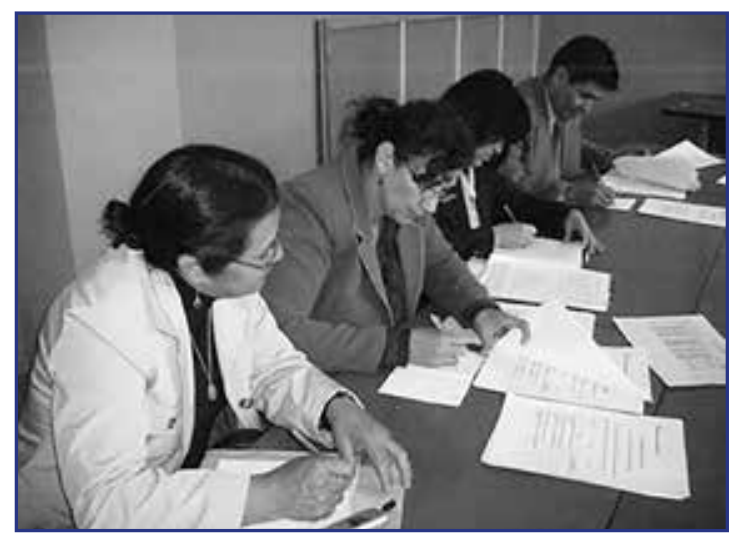

Figura 4. Elaboración de instrumentos de Autoevaluación.

Los planes de mejora constituyen la etapa final como se muestra en la Figura 5 en la que los estudiantes de una escuela decidieron mejorar los ambientes físicos de manera participativa.

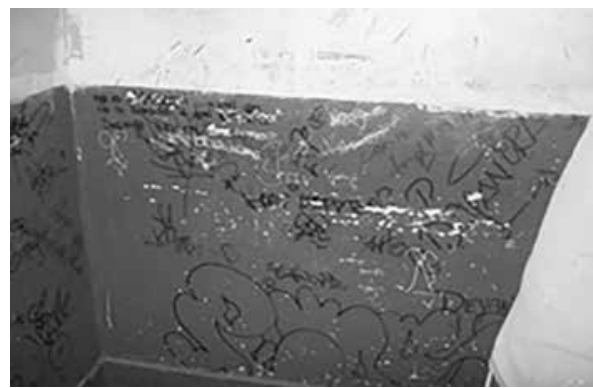

A. Situación problemática

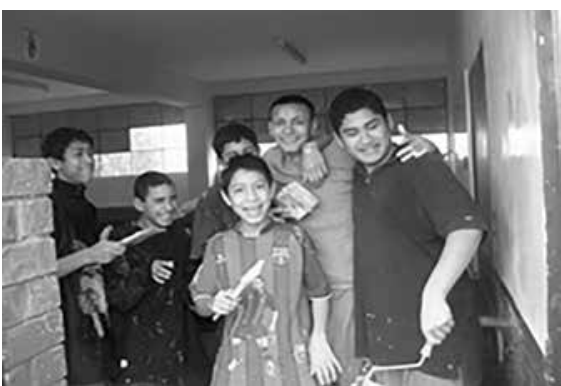

B. Acciones de mejora

Figura 5. Implementación de planes de mejora

Al finalizar el proceso se realizó un encuentro de Buenas Prácticas en Autoevaluación entre todos los participantes (Figura 8). 


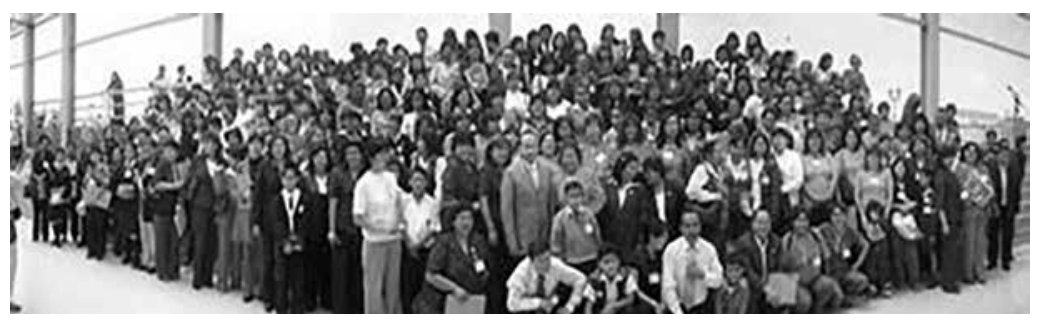

Figura 6. Encuentro de Buenas Prácticas de Autoevaluación.

\subsubsection{Las escuelas seleccionadas}

La selección de escuelas la hizo la Dirección Regional de Educación del Callao en base a dos criterios: interés de los directivos y escuelas que atravesaban dificultades por lo que requerían un mayor apoyo (Tabla 4). Más adelante se verá que no fueron criterios idóneos para iniciar un proceso de cambio institucional.

Tabla 4. Distribución de instituciones educativas participantes al proyecto

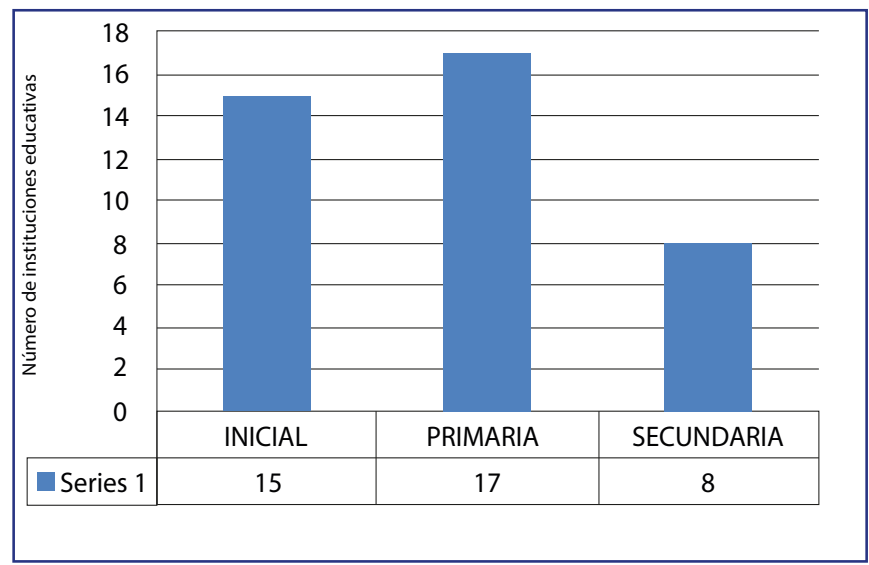

\subsubsection{La imagen emergida de la escuela}

La aplicación del Perfil de Autoevaluación tuvo como resultado el gráfico que se muestra en la Figura 7. Tres fueron los tópicos en los que más escuelas coincidieron en la elección de la temática para realizar al autoevaluación: la escuela como espacio de desarrollo personal y social, el apoyo que se realiza a los estudiantes con problemas de aprendizaje y las relaciones de la escuela con la familia. 


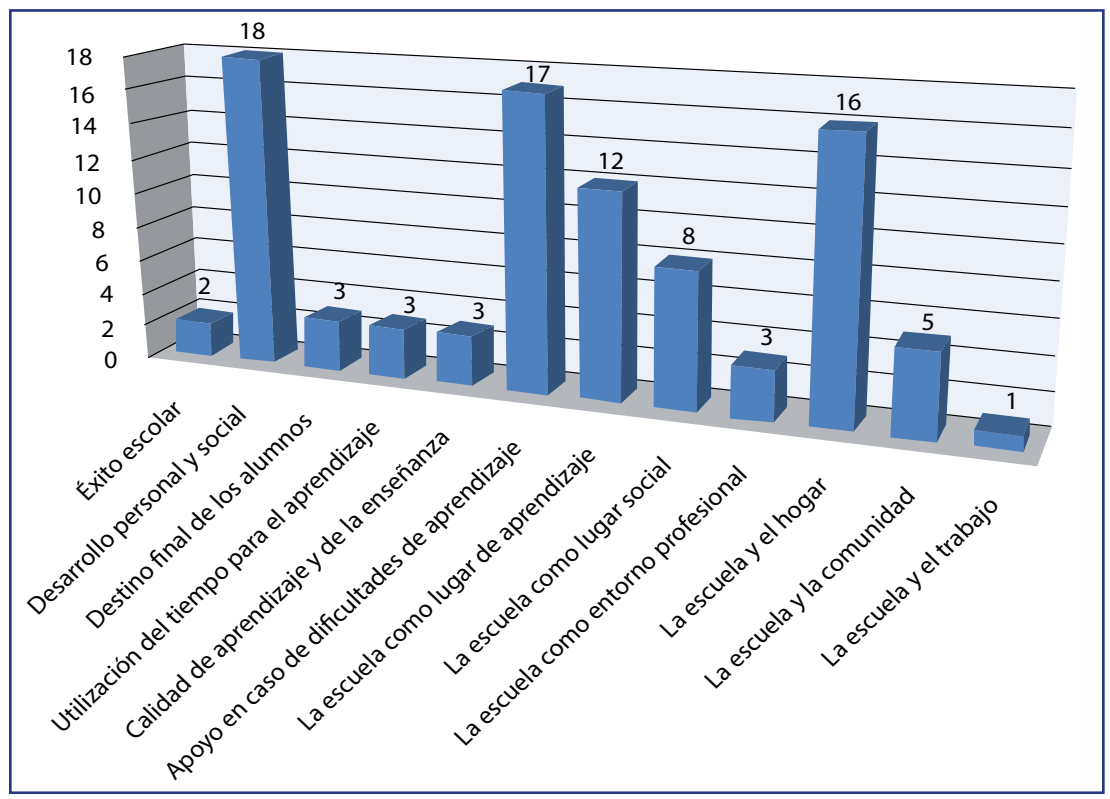

Figura 7. Áreas seleccionadas para ser evaluadas

Los objetivos del estudio fueron comprender las expectativas y opiniones de los principales actores acerca de los elementos del proyecto de autoevaluación. En segundo lugar se buscó analizar el grado de comprensión de los elementos del proyecto y, por último, las reacciones y percepciones frente al incentivo económico y el acompañante escolar.

\section{METOdOLOGÍA}

Debido al número de escuelas participantes se aplicaron encuestas y revisión documental.

\subsection{Población, muestra y muestreo}

\section{Población del estudio}

La población es el conjunto de todos los elementos que son objeto de un estudio. El objeto de estudio son todos los docentes pertenecientes a las escuelas participantes al proyecto de autoevaluación escolar durante el año 2007. 


\section{Muestra}

La muestra es un subconjunto, extraído de la población (mediante técnicas de muestreo), cuyo estudio sirve para inferir características de toda la población. En este caso se ha asumido un muestreo no probabilístico de tipo intencional. Se ha elegido a los miembros de los comités de autoevaluación por las siguientes razones:

1. Debido a que el proyecto inició entre los meses de agosto a septiembre la participación de toda la comunidad educativa se redujo, en algunos casos, a los talleres participativos. Entonces, los que más han intervenido han sido los miembros del comité de autoevaluación.

2. El comité de autoevaluación dirigió todo el proyecto en la escuela con el apoyo del acompañante; estos miembros han vivido de manera cotidiana la participación en el proceso por lo que su opinión acerca del mismo es más amplia y fundamentada.

28 instituciones educativas participaron los 5 meses de trabajo del proyecto, en los que los miembros de los comités de autoevaluación eran un promedio de 6 personas. Lo que hace un total de 168 encuestados. La devolución de las mismas fue de 124 es decir al $76 \%$, pero al $100 \%$ de escuelas (Tabla 5).

Tabla 5

Distribución de los encuestados según sexo

\begin{tabular}{lcccc}
\hline & Docentes & Padres & Alumnos & Total \\
Mujeres & 82 & 10 & 8 & 100 \\
Hombres & 19 & 1 & 4 & 24 \\
\hline Total & 101 & 11 & 12 & 124 \\
\hline
\end{tabular}

\subsection{Técnicas e instrumentos utilizados}

Por las características del objeto de estudio el trabajo resulta consistente con un diseño de tipo encuesta (Creswell, 2008, p. 29), dado que nos interesa conocer la percepción de los actores respecto de un proceso de cambio. Así mismo usamos encuesta para poder abarcar un mayor número de entrevistados. En este caso la opinión de 112 docentes de educación inicial, básica y secundaria. Las preguntas abiertas fueron procesadas por medio de agrupamiento de categorías de significado, puesto que "el mundo del sentido común se encuentra tipificado en categorías 
de significado que permiten reconocer los nuevos fenómenos e incorporarlos a la conciencia del sujeto" (Schutz, 1967). Para la validación de la encuesta se realizó la validación de expertos y validación de caso único (Murillo, 2001). Para la primera se contó con el apoyo del asesor de tesis y para la segunda fueron seis amigos de la calidad seleccionados para responder y revisar la encuesta. Para la aplicación de la encuesta se contactó a cada institución educativa por medio de los amigos de la calidad y se pidió que una reunión del comité de autoevaluación fuera separada sólo para responder a la encuestas. Para evitar un sesgo en la resolución de la encuesta, el aplicador no fue el asesor de la escuela sino otro asignado aleatoriamente. La encuesta fue aplicada durante la primera y segunda semana de abril del 2008. Se realizó también el análisis documental de las bitácoras usadas por cada acompañante. Usando también la técnica agrupamiento de categorías de significado.

\section{RESULTADOS DEL ESTUDIO}

\subsection{Características de la población encuestada}

La mayoría de respondientes a la encuesta han sido mujeres, el 80,56 \% miembros del comité de autoevaluación son mujeres (ver Tabla 8).

\section{Tabla 6}

Porcentaje de encuestados representantes de los diferentes actores

\begin{tabular}{lcccc}
\hline & Docentes & Padres & Alumnos & Total \\
Mujeres & 82 & 10 & 8 & 100 \\
Hombres & 19 & 1 & 4 & 24 \\
\hline Total & 101 & 11 & 12 & 124 \\
\hline
\end{tabular}

Uno de los objetivos del proyecto fue fomentar la participación efectiva de toda la comunidad educativa, expresada en la participación en el comité de calidad. Como muestra la Tabla, 8 la participación de alumnos y padres en la encuesta fue minoritaria. Solo se tiene un $4 \%$ de padres de familia y $5 \%$ de alumnos del total de encuestados. Respecto a las edades de los encuestados, se observa que la mayoría son adultos con edades situadas en el rango de 30 a 50 años (Figura 8). Si bien en las escuelas existe una ingente población de docentes mayores de 50 años, podría asociarse a la mayor apertura de docentes adultos a proyectos de innovación educativa. 


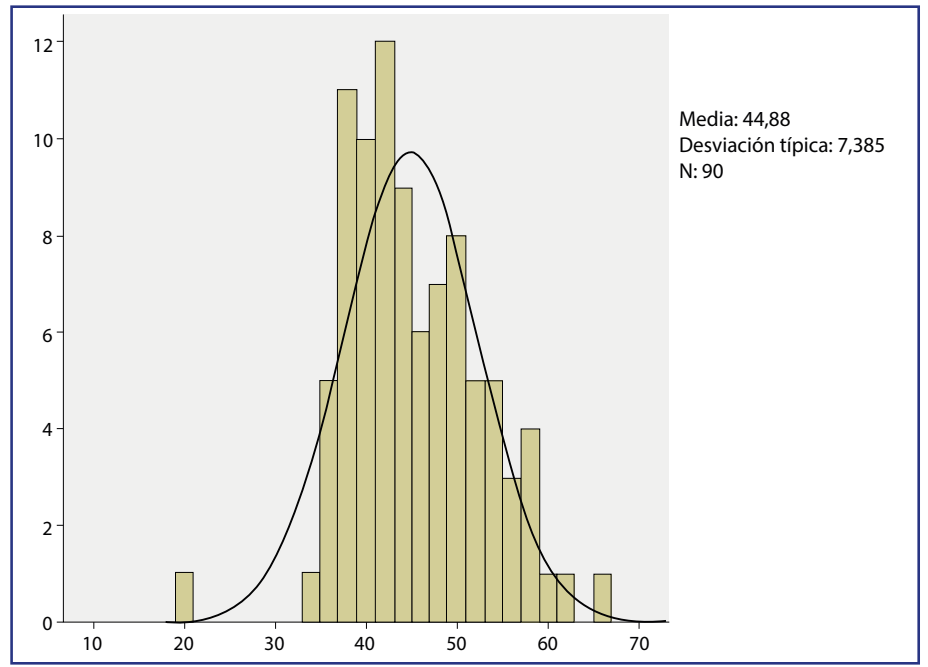

Figura 8. Histograma de las edades de los encuestados.

\subsection{Percepciones respecto a los elementos del proyecto}

Se solicitó al encuestado que responda a preguntas cerradas dicotómicas sobre la realización de cada uno de los objetivos y a preguntas abiertas para realizar un comentario sobre posibles mejoras en la implementación de los mismos. La Figura 9 muestra que, en opinión de los encuestados, el avance de los objetivos del proyecto se realizó en casi la totalidad de instituciones hasta la elaboración y socialización de estándares. Es a partir de los informes por área que se reduce a más de la mitad de logros. En la práctica, solo 3 instituciones educativas presentaron su informe final de autoevaluación, lo que significa que el proceso se terminó; pero, por algunos motivos tuvieron problemas o inconvenientes para la redacción del informe de autoevaluación, entre ellos se podría mencionar el tiempo, dado que la etapa de elaboración de plan de mejora se realizó en diciembre. 


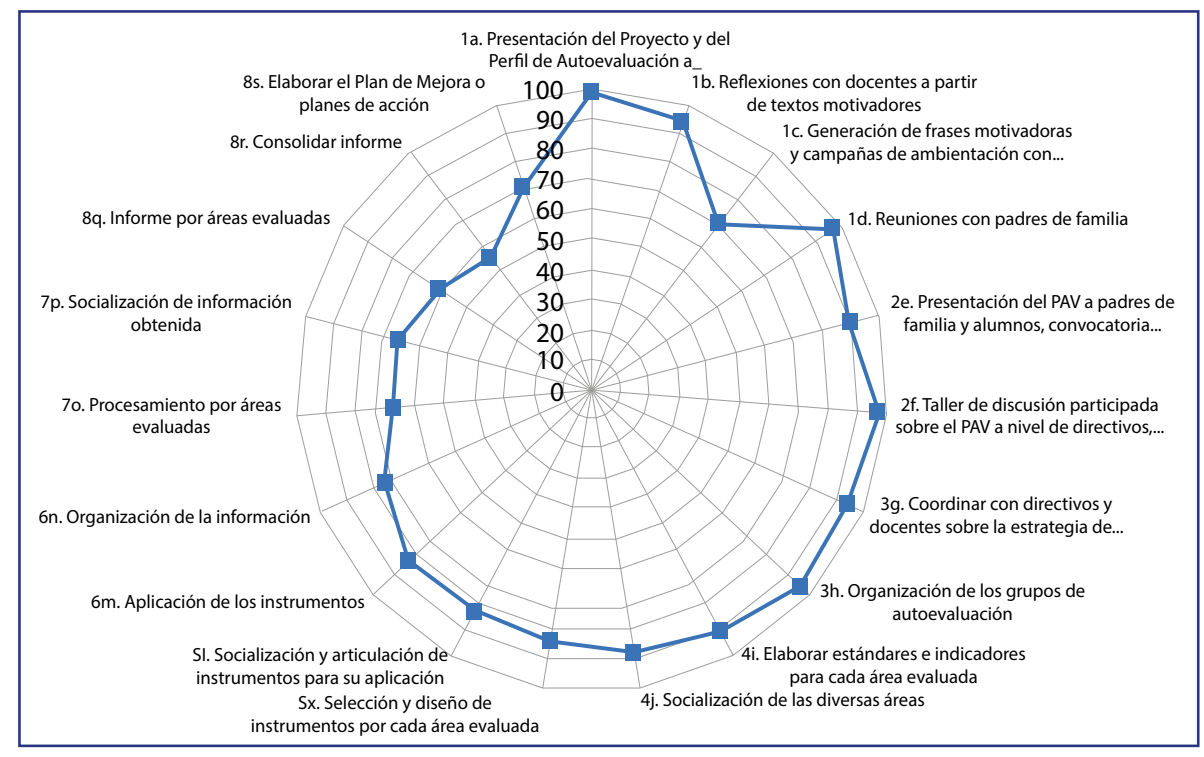

Figura 9. Grado de avance en los objetivos del proyecto.

En la Figura 10 se muestra la pregunta anterior desagregada en padres, profesores y alumnos, esto es qué porcentaje de padres, alumnos o profesores contestaron afirmativamente a cada pregunta. Llama la atención la diversidad de opiniones. Los encuestados coinciden solo en algunas actividades, lo que es explicable dado que la ejecución de las actividades estuvo dada en gran parte por los docentes. Pero, al mismo tiempo, es una muestra del bajo nivel de difusión de las actividades que se realizaron.

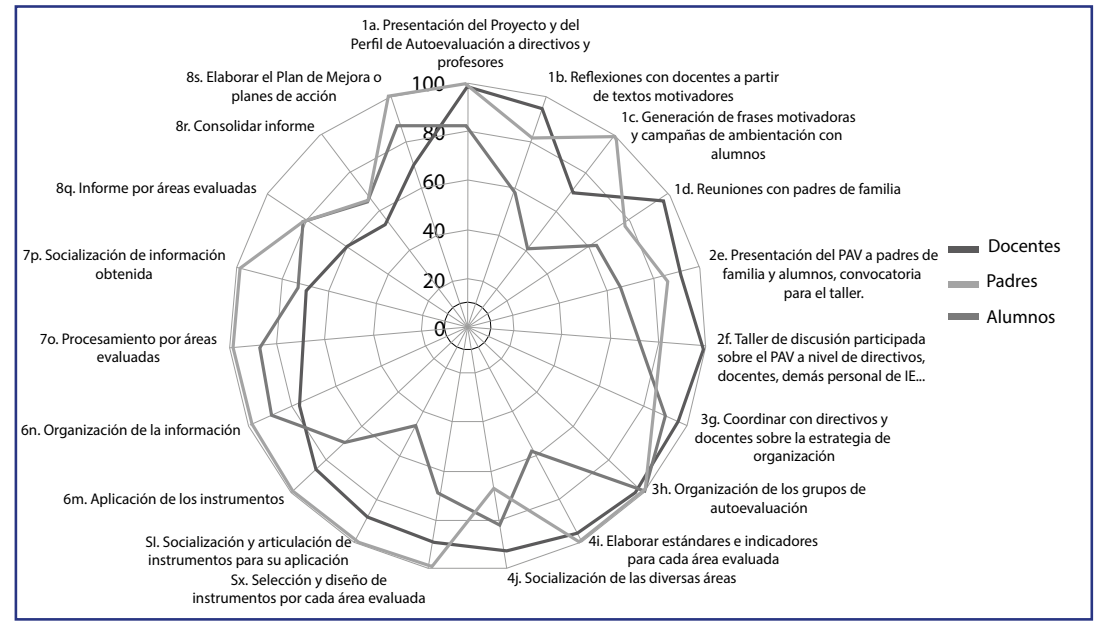

Figura 10. Cumplimiento de los objetivos desde las perspectivas de los actores. 
En elanálisis de las preguntas abiertas, se señala queentre las causas del incumplimiento de actividades, está en primer lugar el "abandono por parte del acompañante" como causa del incumplimiento de algunos objetivos. En efecto, un encargado de escuela, por motivos familiares, dejó de asistir y fue difícil reemplazarlo; dado que, ya se había firmado contrato con el gobierno regional. En segundo lugar, el tiempo escaso para la aplicación de las actividades es un elemento que es mencionado repetidamente por los encuestados. Efectivamente, setiembre y octubre son meses en los que en las escuelas se empieza a sentir el desgaste del avance del año. Otros encuestados cuestionaban en nivel de compromiso logrado en el proceso. Un encuestado afirmó: "Se cumplió, pero la pregunta es icuánto de compromiso se obtuvo?".

Dada la diversidad de escuelas, la constitución y labor del comité de calidad ha sido variada. Algunas escuelas tenían cuatro profesores, por lo que todas eran el comité del autoevaluación, y mientras que en otras los profesores llegaban a ser 94. En el caso de aquellas, la comunicación y trabajo fue más comprometido; y en el caso de estas fue más complicado generar una cohesión en torno al proyecto.

En todo proceso de innovación o introducción de propuestas educativas, el tiempo es un factor muy importante. En el caso de los encuestados, la mayoría tenía poco tiempo de ser parte del comité de autoevaluación. Como se puede ver en la Figura 11, el 32 \% comenzó a ser parte desde agosto.

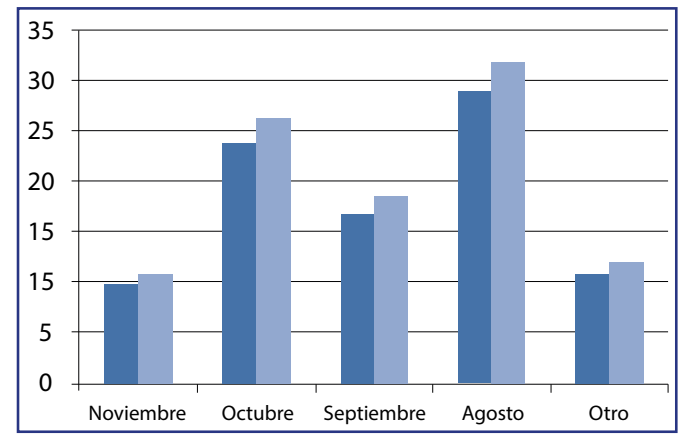

Figura 11. Mes de inicio de participación en el proyecto

Se puede concluir que el trabajo de los comités de calidad ha sido distinto en cada caso dada la diversidad de escuelas, pero en todos los casos ha habido una involucración total de los docentes. Dicha asistencia estaba condicionada al incentivo económico a la participación en los talleres. El incentivo de los padres participantes al comité fue el tener un espacio de opinión y participación en la vida escolar. 
Los talleres de autoevaluación fueron ocasiones en las que, con la participación de la comunidad educativa, se construyó la imagen global del proyecto, los estándares y los indicadores de calidad. Como recomienda Macbeath (2002) un factor importante para el éxito del proyecto y la implementación de los planes de acción es la calidad de la discusión y participación de todos los grupos de interés en los talleres de autoevaluación. La riqueza y uso del Perfil de Autoevaluación (PAE) que se ve en la discusión y dependerá del grado de "representatividad" con el que ha sido construido el grupo mismo. Por esto es importante hacer que se involucren todos y que estén los portavoces de todos los grupos:

Algunos notaron que para los padres y alumnos no siempre era fácil apoyarse con pruebas y argumentaciones; esto es evidente por la falta de experiencia en actividades de este tipo. Pero si se partía reconociendo el estado de carencia informativa para todos se afrontaba la iniciativa como una gran oportunidad de aprendizaje para todos, entonces este se convertía en una experiencia extraordinaria y de una gran eficacia cognoscitiva. (p.96)

Para obtener esta información se presentó un cuadro de doble entrada en el que se debía señalar el grado de asistencia y participación de los actores del proceso. La Tabla 7 muestra que los docentes fueron los que más estuvieron presentes, hecho que es deseable; pero, al mismo tiempo, se evidencia que la presencia de los padres no ha sido permanente hasta en un 67,33\%. Los segundos ausentes han sido los alumnos que llegan a ser parte de algunas reuniones en un $46 \%$.

\section{Tabla 7}

Grado de asistencia de los actores durante los talleres (\%)

\begin{tabular}{lcccc}
\hline & Nunca & $\begin{array}{c}\text { En una } \\
\text { reunión }\end{array}$ & $\begin{array}{c}\text { En algunas } \\
\text { reuniones }\end{array}$ & $\begin{array}{c}\text { En todas las } \\
\text { reuniones }\end{array}$ \\
\hline Padres & 4 & 15 & 67 & 14 \\
Docentes & 1 & 3 & 8 & 88 \\
Alumnos & 20 & 21 & 46 & 13 \\
Administrativos & 37 & 13 & 37 & 14 \\
Director & 3 & 7 & 26 & 64 \\
\hline
\end{tabular}


Tabla 8

Grado de intervención de los actores durante los talleres (\%)

\begin{tabular}{lcccc}
\hline & Nunca & $\begin{array}{c}\text { En una } \\
\text { reunión }\end{array}$ & $\begin{array}{c}\text { En algunas } \\
\text { reuniones }\end{array}$ & $\begin{array}{c}\text { En todas las } \\
\text { reuniones }\end{array}$ \\
\hline Padres & 8 & 18 & 61 & 13 \\
Docentes & 0 & 2 & 11 & 88 \\
Alumnos & 24 & 24 & 39 & 14 \\
Administrativos & 44 & 11 & 35 & 11 \\
Director & 6 & 3 & 23 & 67 \\
\hline
\end{tabular}

Asimismo, en opinión de los encuestados la calidad de la participación ha sido diversa. A la pregunta por el grado de intervención durante los talleres se observa que, en primer lugar, los padres $(60,92 \%)$ han sido los que menos intervenían, seguidos por los alumnos y los administrativos (Tabla 8). Y al parecer de $37 \%$ de los encuestados los que nunca han participado han sido los administrativos. Algunas posibles explicaciones son las siguientes: la ausencia de incentivos económicos para dichos trabajadores y una visión reductiva sobre el cambio escolar, en la que los administrativos no tienen un rol directo o indirecto en la mejora de aprendizajes.

A la pregunta número 1, sobre el proceso de sensibilización, los docentes afirman que los elementos que deben reforzarse en el proyecto son mayor claridad, difusión o comunicación y un mayor apoyo. El factor tiempo se repite mucho, así como, un mayor apoyo con materiales. Efectivamente, la claridad es un elemento muy importante. Argyris y Schon (1978, p. 75) afirman que "la claridad (de los objetivos y de los medios) es un problema constante en el proceso de cambio". Otro problema que se hace presente es la falsa claridad, puesto que "ocurre cuando el cambio se interpreta de forma demasiado simplista; es decir, el cambio propuesto es más profundo que lo que la gente percibe o puede darse cuenta" (Fullan, 2002, p. 104), lo que se discutirá más adelante, estuvo presente de alguna manera en este proyecto.

Los encuestados, en su mayoría, se muestran satisfechos con los talleres. En la Tabla 9, se puede observar que si bien no llegan al extremo de estar "totalmente de acuerdo" al cien por ciento con las opciones planteadas; si se suma los que marcaron las dos gradaciones más altas se llega al $80 \%$ en la mayoría de respuestas. El único caso en el que el mayor porcentaje de opiniones se encuentra en un ítem es cuando se les pregunta si los talleres fueron aburridos. Entonces, se puede decir que los 
talleres fueron útiles, enriquecedores y que se necesitó un mayor tiempo para debatir las temáticas planteadas. Esta pregunta iba acompañada por una pregunta abierta sobre los mismos. Los encuestados manifiestan en su mayoría una opinión positiva de los mismos. En algunos casos mencionan mejorar la dinámica de los mismos.

\section{Tabla 9}

Opiniones respecto a la utilidad de los talleres

\begin{tabular}{lcccccc}
\hline & & $\begin{array}{c}\text { Nada de } \\
\text { acuerdo }\end{array}$ & $\begin{array}{c}\text { Poco de } \\
\text { acuerdo }\end{array}$ & De acuerdo & $\begin{array}{c}\text { Totalmente } \\
\text { de acuerdo }\end{array}$ & Total \\
\hline Útiles & frec. & 2 & 5 & 43 & 41 & 91 \\
Interesantes & $\%$ & 2.2 & 5.49 & 47.25 & 45.05 & 100 \\
& frec. & 2 & 9 & 45 & 40 & 96 \\
Bien organizados & $\%$ & 2.08 & 9.38 & 46.88 & 41.67 & 100 \\
& frec. & 1 & 21 & 45 & 21 & 88 \\
Enriquecedor & frec. & 1.14 & 23.86 & 51.14 & 23.86 & 100 \\
& $\%$ & 1.15 & 14.94 & 42.53 & 41.38 & 100 \\
Aburridos & frec. & 54 & 17 & 2 & 0 & 73 \\
\multirow{5}{*}{ Debimos tener } & $\%$ & 73.97 & 23.29 & 2.74 & 0 & 100 \\
mas talleres & frec. & 3 & 12 & 34 & 33 & 82 \\
\hline
\end{tabular}

En síntesis, los talleres de autoevaluación, si bien fueron pocos (3 a 4), los realizados con toda la comunidad educativo fueron positivos para la vida escolar.

El acompañamiento externo a procesos de cambio ha demostrado ser un catalizador importante. Los resultados de la encuesta mostraron que la gran parte de docentes ve positivamente la figura del Acompañante. Existe un grado de satisfacción con el acompañante $(86,2 \%)$ por parte de los docentes (Tabla 10). Para evaluarlo se aplicaron cinco preguntas cerradas politómicas para evaluar el desempeño y aceptación del acompañante en las escuelas, con una pregunta abierta. 
Tabla 10

Opiniones frente al amigo crítico

\begin{tabular}{|c|c|c|c|c|c|c|}
\hline $\begin{array}{l}\text { Amigo } \\
\text { de la } \\
\text { calidad }\end{array}$ & Estadístico & $\begin{array}{c}\text { Muy } \\
\text { satisfecho o } \\
\text { satisfecho } \\
\text { con el } \\
\text { trabajo del } \\
\text { A.C. }\end{array}$ & $\begin{array}{l}\text { Visitaba } \\
\text { el colegio } \\
1 \text { o más } \\
\text { de } 1 \text { vez } \\
\text { o por } \\
\text { semana }\end{array}$ & $\begin{array}{l}\text { Reuniones } \\
\text { eran dirigidas } \\
\text { generalmente } \\
\text { por el A.C. }\end{array}$ & $\begin{array}{c}\text { La } \\
\text { evaluación } \\
\text { hubiese } \\
\text { avanzado } \\
\text { más rápido } \\
\text { con otro A.C. }\end{array}$ & $\begin{array}{c}\text { El A.C. } \\
\text { demostraba } \\
\text { dominio del } \\
\text { tema }\end{array}$ \\
\hline \multirow{2}{*}{1} & $\%$ & 95 & 94.7 & 95 & 17.6 & 100 \\
\hline & d.s. & -0.22 & -0.23 & -0.22 & -0.39 & 0 \\
\hline \multirow{2}{*}{2} & $\%$ & 100 & 90.9 & 81.8 & 10 & 100 \\
\hline & d.s. & 0 & -30.15 & -40.45 & -31.62 & 0 \\
\hline \multirow{2}{*}{3} & $\%$ & 100 & 85.7 & 100 & 0 & 85.7 \\
\hline & d.s. & 0 & -37.8 & 0 & 0 & -37.8 \\
\hline \multirow{2}{*}{4} & $\%$ & 92.9 & 41.7 & 100 & 0 & 92.9 \\
\hline & d.s. & -26.73 & -51.49 & 0 & 0 & -26.73 \\
\hline \multirow{2}{*}{5} & $\%$ & 68.4 & 68.4 & 83.3 & 27.8 & 88.2 \\
\hline & d.s. & -47.76 & -47.76 & -38.35 & -46.09 & -33.21 \\
\hline \multirow{2}{*}{6} & $\%$ & 81.3 & 43.8 & 93.8 & 7.1 & 100 \\
\hline & d.s. & -40.31 & -51.23 & -25 & -26.73 & 0 \\
\hline \multirow{2}{*}{7} & $\%$ & 71.4 & 85.7 & 85.7 & 20 & 100 \\
\hline & d.s. & -48.8 & -37.8 & -37.8 & -44.72 & 0 \\
\hline \multirow{2}{*}{8} & $\%$ & 90.9 & 72.7 & 81.8 & 0 & 100 \\
\hline & d.s. & -30.15 & -46.71 & -40.45 & 0 & 0 \\
\hline \multirow{2}{*}{9} & $\%$ & 75 & 100 & 100 & 0 & 75 \\
\hline & d.s. & -50 & 0 & 0 & 0 & -50 \\
\hline \multirow{3}{*}{ Total } & $\%$ & 86.2 & 72.6 & 90.6 & 11.3 & 95.2 \\
\hline & d.s. & -34.61 & -44.79 & -29.37 & -31.87 & -21.4 \\
\hline & $\mathrm{N}$ & 109 & 106 & 106 & 97 & 105 \\
\hline
\end{tabular}

Solo el $11 \%$ hubiese estado de acuerdo con trabajar con otro acompañante. Pero desagregando llegamos a tener un caso en el que $27 \%$ hubiesen preferido a otro asesor como se puede apreciar en la Figura 12. Respecto al dominio de tema $95.2 \%$ cree que el Acompañante dominaba el tema que exponía, pero se da un caso en el que 75\% está de acuerdo con lo afirmado. Esta situación se da, según los docentes encuestados, sobre todo por la irresponsabilidad e impuntualidad del mismo, al no 
asistir en los momentos y horas acordadas, lo que generó en alguna escuela un descontento para con el Acompañante.

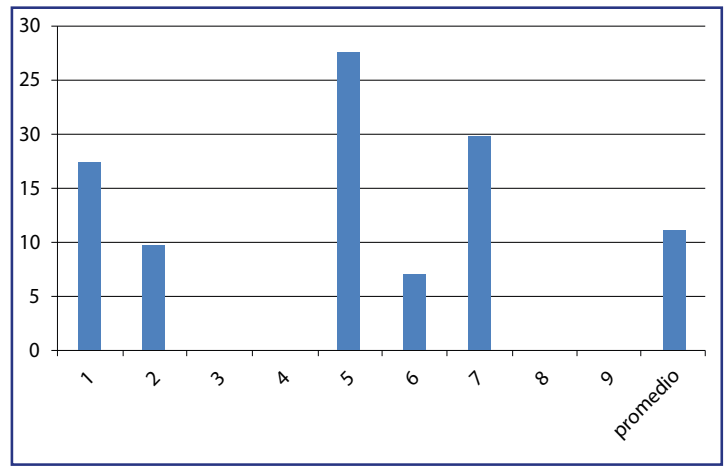

Figura 12. Grado de acuerdo frente a la afirmación "La Autoevaluación hubiese avanzado más si hubiésemos tenido otro acompañante"

Existe un porcentaje relativamente significativo (11\%) que afirma que el Acompañante fue uno de los que impidió el avance adecuado del proyecto desde la perspectiva de los encuestados. La Tabla 11 muestra la opinión del acompañante de la perspectiva de los tres actores. El grado de satisfacción de los alumnos en un $66 \%$. Y en la pregunta por la posibilidad de tener otro acompañante, la mitad de padres y alumnos está de acuerdo con dicha afirmación (57 y $50 \%$ respectivamente). Estas respuestas se pueden explicar por el menor grado de relaciones con los padres y los estudiantes.

Tabla 11.

Porcentaje de encuestados que estuvo de acuerdo con las siguientes afirmaciones por Rol en la I.E.

\begin{tabular}{lcccc}
\hline & Docentes & Padres & Alumnos & Total \\
& 84.2 & 88.9 & 66.7 & 82.8 \\
Muy satisfecho o satisfecho con el trabajo del A.C. & $(36.7)$ & $(33.3)$ & $(49.2)$ & $(37.9)$ \\
& 70.4 & 70.0 & 83.3 & 71.7 \\
Visitaba el colegio 1 o más de 1 vez o por semana & $(45.9)$ & $(48.3)$ & $(38.9)$ & $(45.3)$ \\
& 89.8 & 55.6 & 60.0 & 84.6 \\
Reuniones eran dirigidas generalmente por el A.C. & $(30.4)$ & $(52.7)$ & $(51.6)$ & $(36.2)$ \\
La autoevaluación hubiese avanzado más rápido & 13.5 & 57.1 & 50.0 & 20.4 \\
con otro A.C. & $(34.3)$ & $(53.5)$ & $(52.2)$ & $(40.5)$ \\
& 93.8 & 100.0 & 100.0 & 94.8 \\
El A.C. demostraba dominio del tema & $(24.2)$ & $(0.0)$ & $(0.0)$ & $(22.3)$
\end{tabular}


Del análisis de la pregunta abierta sobre el grado de satisfacción con el trabajo realizado por el Acompañante, existe un mayoritario consenso de la acción positiva del mismo. Salvo en los casos antes mencionados de deserción de un Acompañante. Así mismo, los participantes valoran mucho las cualidades humanas del mismo, como son la paciencia, la comprensión, la involucración al punto de quedarse más allá de la hora pactada. Y desde el punto de vista profesional valoran la capacidad de explicación, de exhortar y movilizar emociones para el logro de los objetivos del proceso. Pero en general los participantes apelan o valoran más características personales que profesionales. Lo que confirma la importancia de la gestión de las emociones en la escuela (Labaree, 1997b).

El rol de la dirección escolar en procesos de cambio es clave (Fullan, 2008; Fundación Wallace, 2012; Geijseln, Sleegers, Stoeln, \& KrÃgern, 2009; Leithwood, Louis, Anderson, \& Wahlstrom, 2004). El proceso de autoevaluación es un proceso de cambio en las prácticas profesionales de los docentes y un cambio de concepción en la manera de gestionar una institución educativa, por lo que se aplica la opinión de los especialistas. Es importante la asistencia del director a las reuniones de coordinación de la innovación a implementar, dependiendo de ello el futuro de dicha implementación (Fullan, 2007). Es por esa razón, que se elaboró un ítem para evaluar la asistencia de los directores y el grado de participación en los talleres (Figura 13).

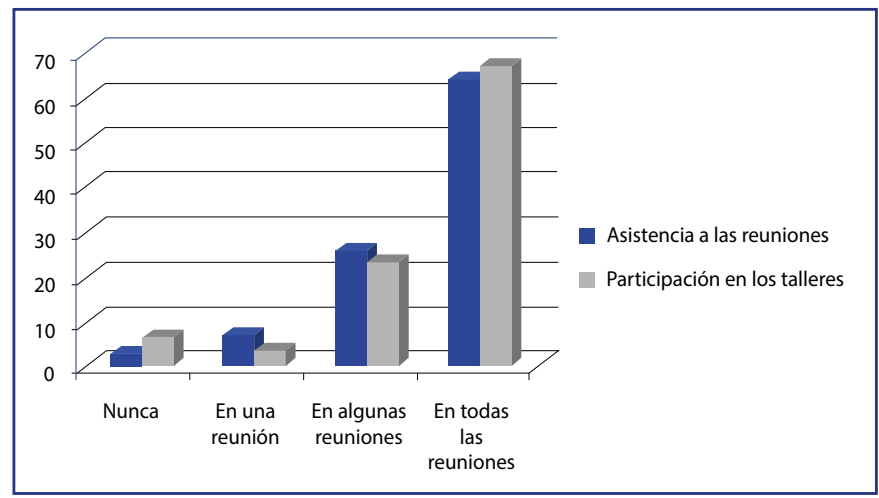

Figura 13. Asistencia y participación de los directivos a los talleres y reuniones

Los directores estuvieron presentes en la mayoría de reuniones del proyecto. Como se puede observar un grupo de $26 \%$ y $22 \%$ respectivamente de profesores opina que el director sólo estuvo presente en algunas reuniones. Lo que significa un factor positivo para la implementación del proyecto. Se podría afirmar, en consecuencia, que el avance de las actividades del proyecto hubiese sido menor sin la presencia de éstos. 
La mitad de los docentes encuestados cree que se han realizado las acciones motivados en buena parte por el incentivo económico (Figura 14).

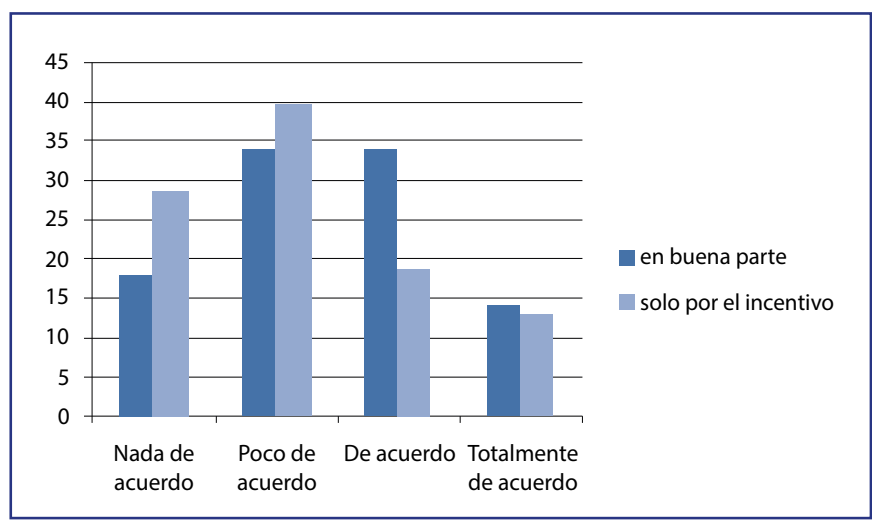

Figura 14. Grado de motivación del incentivo económico

Respecto a la manera de otorgar los incentivos la mayoría de docentes cree que no fue complicada (Figura 15), puesto que en la mayoría de escuelas los incentivos se recibían por la participación en reuniones semanales.

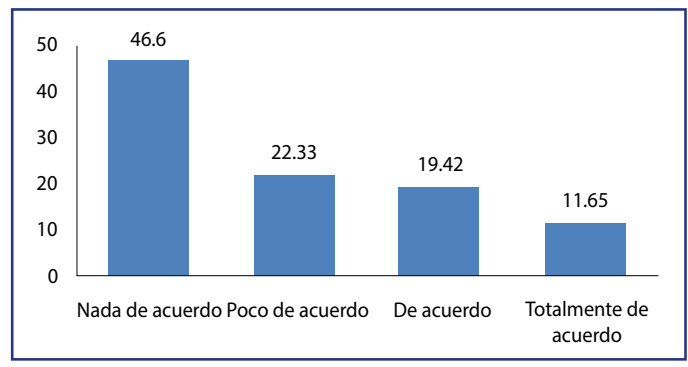

Figura 15. Complicaciones de medición para el otorgamiento del incentivo

En la pregunta abierta acerca de cómo mejorar la entrega del incentivo aparecen varios comentarios. Algunos en la línea de la motivación extrínseca y otros convencidos que la motivación intrínseca es el mejor motor de cambio. Las siguientes afirmaciones apoyan la motivación extrínseca:

"Incentivo debería ser mayor de acuerdo con el logro" (Encuestado 13).

"Que deben dar el incentivo de acuerdo al trabajo realizado y de manera puntual. Grupo que trabaja más debe tener mayor incentivo" (Encuestado 65) 
"Soy una de las personas convencidas de que dicho incentivo económico debe proporcionarse tras la obtención de productos objetivos" (Encuestado 71).

Otros comentarios van en la línea de la expansión a todo el staff educativo de los incentivos (administrativos y auxiliares). Otros plantean que se aumente el monto entregado. Así mismo recomiendan la puntualidad de la entrega hecho que no ocurrió por las razones más arriba explicadas.

Los que van en la línea de la motivación intrínseca afirman lo siguiente: "No hay mayor incentivo que el buscar la mejora y el crecimiento de nuestra escuela, y el que nos den las herramientas e instrumentos necesarios para elevar la calidad de nuestra institución" (Encuestado 21).

Dicho comentario acentúa una motivación intrínseca en la que el móvil por el cambio está en el aprendizaje mismo de los alumnos (Greeno, Collins, Resnick, Berliner, \& Calfee, 2006).

Otros proponen generar diversos tipos de incentivos como los siguientes:

"Que la docente que toma el interés y dedicación a su labor en el nivel inicial, sea considerada con incentivos no solamente económicos sino también en estudios, información como libros, foros, etc." (Encuestado 59).

Además de: "textos, refrigerios, capacitaciones, paseos, jornadas" (Encuestado 1), "capacitaciones, diplomados" (Encuestado 2), "un certificado de haber participado" (Encuestado 3), "separatas, videos por internet" Encuestado 7), "viaje a otros países que han tenido éxito cursos de capacitación con empresas de resultados exitosos" (Encuestado 8).

De las afirmaciones anteriores, se evidencia el grado de valoración que se le da al incentivo económico y a la certificación (Labaree, 1997a). Así mismo, buena parte de los encuestados reconoce el interaprendizaje o aprendizaje entre pares como un factor que ayuda a la mejora y que incentiva el éxito.

Para cruzar respuestas, se creó un ítem que decía lo siguiente: "una escuela participó del proyecto sin incentivos económicos, ¿qué opinión le merece?" La mayoría felicitó la iniciativa aduciendo lo loable del compromiso y amor a los niños y a la escuela. Aquellos que opinaron de manera negativa utilizaron argumentos de tipo familiar y económico ("Tenemos familia", "los sueldos docentes son bajos", etc.). 
Por lo que se puede concluir que fue un elemento importante para la implementación del proyecto. Hubiera sido más enriquecedor otorgar fondos para la escuela para apoyar las iniciativas, fruto del proceso de autoevaluación.

\subsection{Experiencia y opiniones generales acerca del proyecto}

El presente acápite está divido en tres partes: un análisis costo-beneficio del proyecto en opinión de los actores, los roles más importantes para la implementación del mismo y recomendaciones finales para la mejora del mismo. Se aplicaron nueve ítems para recoger esta información.

El análisis de las preguntas dicotómicas cerradas muestra que para el 69\% de los encuestados es la primera vez que se trabaja con todos los interesados, a pesar de que, según la normativa vigente existen numerosos espacios de participación, muchos de ellos con un funcionamiento irregular como es el caso del Consejo Educativo Institucional (CONEI). Un 59\% de los encuestados muestra su acuerdo con la afirmación "Por fin, ha llegado el momento de dejar de engañarnos" (Tabla 12). Esto significa que se reconoce que en la gran mayoría de las escuelas se vivía o se vive aún muchos espacios de cuidar imagen o de tratar de "llevar la fiesta en paz", es decir, no reconocer una realidad en crisis por la cual atraviesa la escuela pública hace mucho tiempo. Por lo que es posible afirmar que el proyecto ha contribuido a cuestionar la "gramática escolar" existente (Tyack \& Cuban, 1997). Esto entendido como un primer paso para dar solución al problema del desacoplamiento docente (Benavides, Rodrich, \& Mena, 2009; Coburn, 2004). Asimismo, se observa que un $31.7 \%$ piensa que no estaba dando resultados.

Tabla 12.

Porcentaje de encuestados que están de acuerdo con las siguientes opiniones.

\begin{tabular}{lcccc}
\hline & $\mathrm{N}$ & Hombres & Mujeres & Total \\
\hline P33. "Me gusta el proyecto porque es la primera & & 81 & 65.9 & 69.5 \\
vez que veo a todos juntarse en un mismo saco" & 105 & -40.2 & -47.7 & -46.3 \\
P34. "Por fin, ha llegado el momento de dejar de & & 57.9 & 57.5 & 58.5 \\
engañarnos" & 94 & -50.7 & -49.8 & -49.5 \\
P35. "Siento que EA no da resultados & & 30 & 31.7 & 31.7 \\
& 101 & -47 & -46.8 & -46.8 \\
\hline
\end{tabular}


En la Figura 16 se observa que la gran mayoría de los encuestados piensa que el proyecto ha sido útil para la vida de la escuela. Cerca del $87 \%$ piensa que la participación en el proyecto ha sido útil.

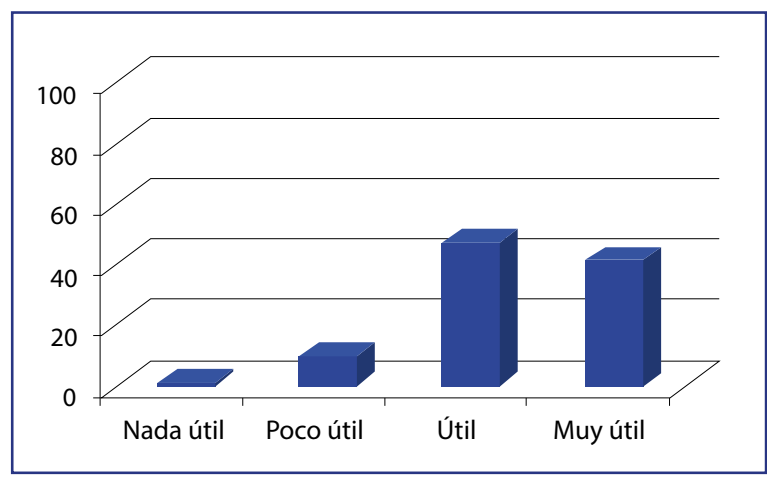

Figura 16. Opinión respecto a la pregunta "¿Cómo consideró la experiencia de participar en el proyecto?"

La mayoría de los encuestados afirma haber aprendido durante estos meses (96\%), mientras que, una gran mayoría estaría de acuerdo en participar en un proceso similar; pero considera que hubiera sido interesante añadir "sin incentivos" para eliminar dicha variable interviniente.

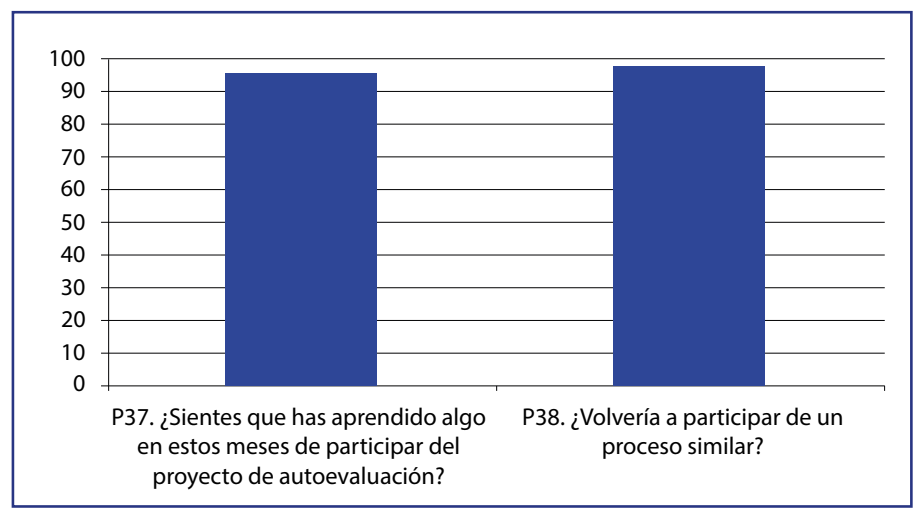

Figura 17. Actitud frente al proyecto

Se hizo la siguiente pregunta abierta a los encuestados: "¿Qué ha significado para ti Escuelas de Avanzada? ¿Qué cambios ha habido en tu escuela debido al proyecto?". 
Para la primera pregunta, la mayoría respondió que la participación fue muy provechosa tanto para el crecimiento personal como para el crecimiento institucional: algunos lo han visto como "un cambio y un nuevo sistema de hacer proyectos de la institución educativa y la comunidad" (E 84). Es decir, con un aporte importante para la realización de innovaciones en la escuela y con la comunidad.

Algunos comentarios sobre las vivencias personales: "para mí ha sido una forma de mejorar mi práctica docente a través de la autoevaluación a la vez que se mejoran las relaciones humanas" (E 101). Aquí se puede observar un efecto en la mejora del clima institucional.

Así mismo, el proyecto permitió "cambiar conceptos errados sobre la educación y empezar a trabajar los profesores, administrativos, alumnos y padres de familia en conjunto" (Encuestado 86).

Un encuestado afirmó que el proyecto fue "Una oportunidad de autoanálisis, el problema es que estamos tan apremiados con participar en tantos eventos, que no se sabe, si avanzamos o no. Esto nos permite hacer un alto en nuestro trabajo y permitirnos reflexionar para avanzar" (Encuestado 37). Esta realidad hace referencia a la sobrecarga de actividades que tiene la vida en las escuelas, Brick acuñó el término de fenómeno del árbol de Navidad (Brick 1996 citado en Fullan, 2002).

\subsubsection{Roles que más influyeron en la implementación del proyecto}

Para identificar los roles que más influyeron en la implementación del proyecto se aplicaron dos ítems. Uno con la afirmación: "El proyecto hubiese ido mejor con más compromiso de todos" y otro consultaba sobre el rol que tuvo más utilidad para la implementación del mismo. La mayoría de encuestados afirma que es necesario la presencia de los padres y de la coordinación general para la implementación del proyecto (Figura 18). 


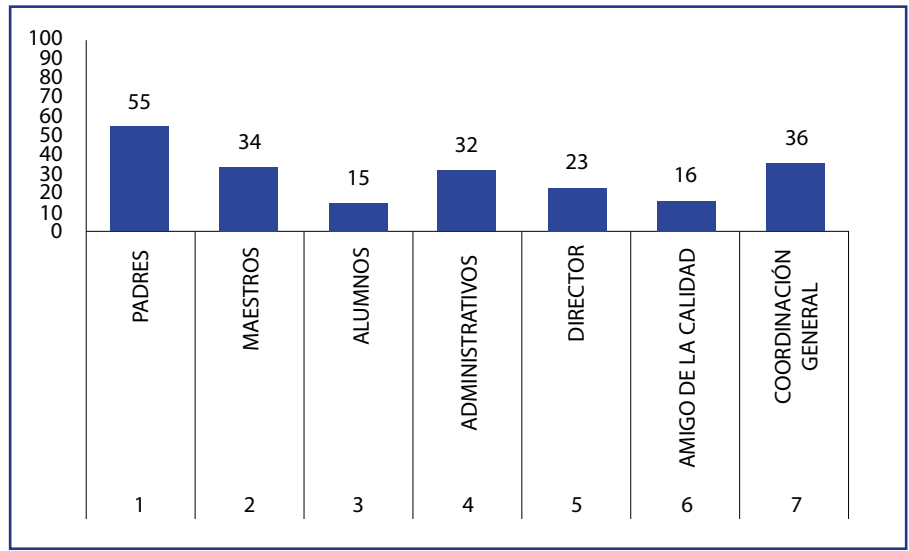

Figura 18. Roles que más influyeron en la implementación

A la pregunta abierta que complementaba, los encuestados afirmaron que hubiera sido útil la participación de Gobierno Regional, la UGEL e instituciones aliadas de la escuela como la parroquia, comisaría, bomberos, etc. En el proyecto Bridges Across the Boundaries, se invitó a participar en los talleres de autoevaluación a dichas instituciones. En este proyecto se dio esta indicación, pero en muchos casos no logró realizarse. Se puede concluir que los encuestados reconocen la importancia de involucrar a todos los grupos interesados o que tienen relación con la escuela a nivel horizontal y vertical para poder implementar adecuadamente esta propuesta. Además, el rol que fue más útil fue el de los docentes (88\%), seguidos por la participación del acompañante (82\%), los alumnos (61\%) y los padres de familia (57\%). La presencia del acompañante tiene un peso muy cercano al de los docentes.

\section{CONCLUSIONES}

En términos generales, los participantes en este estudio manifestaron una actitud favorable al proceso de autoevaluación experimentado. Es necesario aclarar, que el acompañante fue un elemento importante para el logro de los objetivos. Ejerció un rol mediador y de sana presión para el avance del mismo. Queda aún investigar cuánto influye en el logro de resultados el sexo, la raza y la edad del acompañante, así como sus conocimientos personales y el tiempo de acompañamiento ¿Qué niveles de amistad crítica se pueden desarrollar dentro de la escuela (entre maestros) y entre escuelas, aprovechando al máximo los conocimientos y experiencias de los docentes? Lo que sí queda claro es que la escuela vive una situación de aislamiento, que genera 
un "hambre de amistad", tiene una necesidad de "amigos", llámeseles asesores o acompañantes externos. Y, como se vio en esta investigación, el acompañante no nace, es necesario formarlo. Es un rol bastante complejo por lo que se requiere de la implementación de políticas públicas para la formación de asesores escolares de diverso tipo pedagógicos, de gestión organización, y de liderazgo educativo. Distintos aspectos educativos, pero con un solo fin. En el proyecto se optó por contratar a docentes de escuela pública con experiencia en gestión educativa, por lo que tuvo un peso especial el anhelo de justicia social de los mismos, para así poder renovar a las escuelas en su fin transformador de la realidad. La complicación se encontró en que los docentes nombrados solo podían pedir permiso hasta un año. En este proyecto, el $90 \%$ eran nombrados del Estado, como fueron algunos meses el primer año y luego el segundo. Los docentes pidieron permiso cinco meses el primer año y 6 el segundo. Un tercer año de acompañamiento hubiera sido inviable con las mismas personas, perdiendo por lo tanto el capital humano y social desarrollado en los mismos para el acompañamiento escolar.

Se deja entrever que existe una evidencia, relativamente significativa, que otorgar incentivos de tipo monetario genera más complicaciones que beneficios; pero al mismo tiempo son necesarios para el docente. Lo descubierto deja ideas para generar nuevos tipos de incentivos como lo proponen los mismos maestros en su gran mayoría. Estos dejan en claro que un verdadero incentivo es algo que potencie el aprendizaje profesional. En este proyecto la concepción no fue trabajar incentivos en función a resultados; sino, utilizar los incentivos como compensación por los tiempos, recursos y gastos diversos ocasionados por el proyecto. Para algunos autores es importante otorgar apoyos económicos, pero no individualmente sino para cada escuela o una red, para que puedan implementar pequeños proyectos de innovación. También se mencionan experiencias con fondos pequeños para redes escolares, con autonomía total para su uso, sin la intervención del Estado ( "A net without nanny") (Hargreaves, 2009, p. 34). En suma, es importante generar políticas que desarrollen apoyos monetarios a las escuelas, pero de manera grupal, para financiar ideas innovadoras o de mejora educativa. Como es el caso de las Redes de Mejoramiento Escolar (RME) impulsadas desde el año 2015 por el División de Educación General del Ministerio de Educación de Chile (MINEDUC, 2017).

Asimismo, se evidenció la importancia del rol del director en los procesos de cambio. Si el director no comprende la propuesta o no está comprometido con ella, puede obstaculizar la implementación de la misma. Por otro lado, se comprobó que la escuela se ve sometida a muchas fuerzas, todas ellas con intenciones de mejora, 
pero no logra una "coherencia programática" (Newmann, King, \& Youngs, 2000) que se define como el grado en el que los programas escolares para los estudiantes y aprendizajes del staff son coordinados, y centrados en objetivos de aprendizaje claros y sostenidos por un periodo de tiempo. Se observó que las escuelas que formaron parte del proyecto se encontraban sobrecargadas de múltiples proyectos.

Finalmente, se plantean dos reflexiones finales de este estudio. La primera es que la autoevaluación institucional es un medio efectivo para la mejora de la escuela, sin embargo queda aún mucho por aprender sobre cómo generar dichos procesos de manera efectiva adaptándolos a la diversidad de realidades y contextos en los que se desarrolla una institución educativa. En segundo lugar, se ha confirmado lo que menciona la literatura de cambio escolar sobre la calidad y claridad de la innovación para una implementación eficaz. La claridad ha jugado un rol importante en este proceso de autoevaluación.

\section{REFERENCIAS}

Ainscow, M., \& Southworth, G. (1996). School Improvement: A Study of the Roles of Leaders and External Consultants. School Effectiveness and School Improvement, 7(3), 229-251.

Alvik, T. (1996). School Self-evaluation: A Whole School Approach. CIDREE, Dundee.

Argyris, C., \& Schon, D. (1978). Organizational learning: A theory of action perspective.: Addison Wesley, New York.

Bambino, D. (2002). Critical friends. Educational Leadership, 59(6), 25-27.

Baudelot, C., \& Leclercq, F. (2005). Los efectos de la educación. Buenos Aires: Del estante.

Benavides, M., Rodrich, H., \& Mena, M. (2009). Niveles de acoplamiento y desacoplamiento en la relación familia-escuela en contextos rurales: el caso de una muestra de familias de Quispicanchis, Cusco. Revista Peruana de Investigación Educativa, 1(1). 
Brotto, F. (2011). Findings in translation: negotiating and leading learning across borders. In T. Townsend \& J. MacBeath (Eds.), International handbook of leadership for learning (Vol. 25): Springer Verlag.

Castoldi, M. (2001). La scuola sotto esame. Brescia: La Scuola.

Coburn, C. E. (2004). Beyond Decoupling: Rethinking the Relationship Between the Institutional Environment and the Classroom. Sociology of Education, 77(3), 211-244.

Coleman, J. S. (1966). Equality ofEducational Opportunity, New York.

Costa, A. L., \& Kallick, B. (1993). Through the lens of a critical friend. Educational Leadership, 51 (2).

Creemers, B., \& Reezigt, G. (2005). Linking school effectiveness and school improvement: The background and outline of the project. School Effectiveness \& School Improvement, 16 (4), 359-371.

Creswell, J. (2008). Educational research : planning, conducting, and evaluation quantitative and qualitative research (3 ed.). Columbus, $\mathrm{OH}$ : Pearson.

Cuban, L., \& Shipps, D. (2000). Reconstructing the common good in education : coping with intractable American dilemmas. XVI, 283 p.

Dean, J. (1992). Inspecting and Advising: A Handbook for Inspectors, Advisers and Advisory Teachers Editorial Routledge.

Elliott, J. (1981). Action-research: A Framework for Self-evaluation in Schools. Cambridge: Institute of Education.

Fullan, M. (1982). The meaning of educational change. New York: Teachers College, Columbia University.

Fullan, M. (2002). Los nuevos significados del cambio en la educación. Barcelona: Octaedro.

Fullan, M. (2006). Turnaround leadership (1st ed.). San Francisco, CA: Jossey-Bass. 
Fullan, M. (2007). The new meaning of educational change (4th ed.). New York: Teachers College Press.

Fullan, M. (2008). What's worth fighting for in the principalship? New York: Teachers College Press.

Fundación Wallace. (2012). The school principal as leader: guiding schools to better teaching and learning Fundación Wallace, New York.

Geijseln, F., Sleegers, P., Stoeln, R., \& KrÃgern, M. (2009). The Effect of Teacher Psychological and School Organizational and Leadership Factors on Teachers' Professional Learning in Dutch Schools. The Elementary School Journal, 109(4), 406-427.

Greeno, J. G., Collins, A. M., Resnick, L. B., Berliner, D. C., \& Calfee, R. C. (2006). Handbook of educational psychology American Psychological Association y Lawrence Eribaum Associates, Inc., Chicago.

Hanushek, E. (1995). Interpreting recent research on schooling in developing countries. The World Batnk Research Observer, 10(2), 227-246.

Hanushek, E., \& Welch, F. (2006). Handbook of the Economics of Education: Elsevier B.V.

Hanushek, E. A. (2002). The Long Run Importance of School Quality. NBER Working Paper. New York.

Hargreaves, A. (2009). The Fourth Way of Change: Towards an Age of Inspiration and Sustainability. In A. Hargreaves \& M. Fullan (Eds.), Change Wars. Bloominghton: Solution Three.

Hargreaves, A., \& Shirley, D. (2009). The fourth way: The inspiring future for educational change Corwin, Toronto.

Heckman, J. (2011). Hard evidence in soft skills. Chicago: University of Chicago Press.

Heller, H. (1998). The Advisory Service and Consultancy. Management Consultancy in Schools, 117-128. 
Kemmis, S., \& McTaggart, R. (1988). The Action research planner (3rd ed.). Melbourne: Deakin University : distributed by Deakin University Press.

Kemmis, S., \& McTaggart, R. (1988). Como planificar la investigación-acción. Barcelona: Laertes.

Kemmis, S., \& Wilkinson, M. (1998). Participatory action research and the study of practice. Action Research in Practice: Partnerships for Social Justice in Education, 21-36.

Labaree, D. F. (1997a). How To Succeed in School without Really Learning The Credentials Race in American Education, New York.

Labaree, D. F. (1997b). Public goods, private goods: The American struggle over educational goals. American Educational Research Journal, 34(1), 39-81.

Leithwood, K., Louis, K. S., Anderson, S., \& Wahlstrom, K. (2004). How Leadership Influences Student Learning. Review of Research. Wallace Foundation, The, 90.

MacBeath, J. (1998). I Didn't Know He Was III - The Role and Value of the Critical Friend. In L. a. M. Stoll, K. (Ed.), No Quick Fixes: Perspectives on Schools in Difficulties. London: Falmer Press.

Macbeath, J. (1999). Schools must speak fot themselves (pp. 349-366). London: Routledge.

Macbeath, J. (2002). Self-evaluation: What's in it for schools? Londres: Routledge.

MacBeath, J., Schratz, M., Meuret, D., \& Jakobsen, L. (2001). Self-Evaluation in European Schools: A Story of Change. Londres: RoutledgeFalmer.

Macbeath, J., Schratz, M., Meuret, D., \& Jakobsen, L. ( 1999). Evaluating quality in school education A European pilot project. Final report. European commission.

MINEDUC. (2017). Estudio sobre la implementación de las redes de mejoramiento escolar, Ministerio de Educación de Chile.

Murillo, J. (2001). Cuestionarios y escalas de actitudes, Material didáctico, Barcelona. 
Nevo, D. (1995). School-based evaluation: A dialogue for school improvement: Pergamon Oxford, UK.

Newmann, F. M., King, M. B., \& Youngs, P. (2000). Professional Development That Addresses School Capacity: Lessons from Urban Elementary Schools. American Journal of Education, 108(4), 259-299. Recuperado de http://www.jstor.org/ stable/1085442

OEA. (2003). La Autoevaluación como mecanismo de mejoramiento institucional. Lima: OEA.

Psacharopoulos, G., \& Patrinos, H. A. (2004). Returns to investment in education: a further update. Education economics, 12(2), 111-134.

Sammons, P. (1995a). Key Characteristics of Effective Schools: A Review of School Effectiveness Research. England: B \& MBC Distribution Services.

Sammons, P. (1995b). Key characteristics of effective schools: A review of school effectiveness research. B \& MBC Distribution Services, 9 Headlands Business Park, Ringwood, Hants BH24 3PB, England, United Kingdom..

Scheerens, J. (1999). Foundations of School Effectiveness. París: UNESCO.

Scheerens, J. (2000). Improving School Effectiveness. Paris: UNESCO: International Institute for Educational Planning. Fundamentals of Educational Planningseries.

Scheerens, J. (2007). Accountability, autonomy and school effectiveness, Can accountability be seen as a lever of school improvement? Paper presented at the International Congress of School Effectiveness and Improvement 2007.

Scheerens, J., \& Bosker, R. (1997). The Foundations of Educational Effectiveness. Reino Unido: Pergamon.

Scheerens, J., \& Bosker, R. J. (1997). The foundations of educational effectiveness: Pergamon, Holanda.

Schein, E. H. (1997). The concept of "client" from a process consultation perspective. Management, 10(3), 202-216. 
Schutz, A. (1967). The phenomenology of the social world. [Evanston, III.]: Northwestern: University Press.

Stenhouse, L. (1980). Curriculum research and development in action: Heinemann Educational Books. FALTA CIUDAD.

Supovitz, J. (2009). Can high stakes testing leverage educational improvement? Prospects from the last decade of testing and accountability reform. Journal of Educational Change, 10(1), 211-227.

Swaffield, S. (2002). Contextualising the Work of the Critical Friend. Paper presented at the symposium: 'Leadership for Learning: the Cambridge Network'.

Swaffield, S. (2004). Exploring Critical Friendship through Leadership for Learning. Paper presented at the symposium: 'Leadership for Learning: the Cambridge Network'.

Tyack, D. B., \& Cuban, L. (1997). Tinkering Toward Utopia: A Century of Public School Reform Harvard University Press, Cambridge. 